\title{
Specific interactions, structure and properties in segmented polyurethane elastomers
}

\author{
K. Bagdi ${ }^{1,2}$, K. Molnár ${ }^{1,2}$, I. Sajó $^{3}$, B. Pukánszky $y^{1,2^{*}}$ \\ ${ }^{1}$ Laboratory of Plastics and Rubber Technology, Budapest University of Technology and Economics, H-1521 Budapest, \\ P.O. Box 91, Hungary \\ ${ }^{2}$ Institute of Materials Science and Environmental Chemistry, Chemical Research Center, Hungarian Academy of \\ Sciences, H-1525 Budapest, P.O. Box 17, Hungary \\ ${ }^{3}$ Institute of Nanochemistry and Catalysis, Chemical Research Center, Hungarian Academy of Sciences, H-1525 \\ Budapest, P.O. Box 17, Hungary
}

Received 10 September 2010; accepted in revised form 30 November 2010

\begin{abstract}
Two sets of segmented polyurethane (PU) elastomers were prepared from 4,4'-methylenebis(phenyl isocyanate) (MDI), 1,4-butanediol (BD) and a polyester or a polyether polyol, respectively. The molecular mass of both polyols was $1000 \mathrm{~g} / \mathrm{mol}$. The stoichiometric ratio of isocyanate and hydroxyl groups was 1 and the polyol/total diol ratio changed from 0 to 1 in 0.1 steps. One step bulk polymerization was carried out in an internal mixer and the samples were compression molded for testing. The results proved that specific interactions determine the phase structure and properties of these materials. Crystallinity was approximately the same in the two types of polyurethanes and the amount of relaxing soft segments was also similar. The determination of interaction parameters from solvent absorption and differences in glass transition temperatures indicated stronger interaction between hard and soft segments in the polyester than in the polyether polyurethane. Larger transparency of the polyester PU indicated the formation of smaller dispersed particles of the hard phase. The larger number of smaller hard phase units led to the formation of more physical cross-links distributed more evenly in the polymer. These differences in the phase structure of the polymers resulted in stronger strain hardening tendency, larger strength and smaller deformations for the polyester than for the polyether polyurethane.
\end{abstract}

Keywords: biocompatible polymers, polyurethane elastomer, specific interactions, phase separation, physical cross-links

\section{Introduction}

Segmented polyurethanes (PU) are versatile materials used in many segments of life [1]. They are applied as automotive parts, in building and construction, in electrical and engineering applications, in the oil, chemical and food industry and in many other fields $[1,2]$. One of their major application areas is health care, where they are used mainly as medical devices in the form of encapsulants for hollow-fiber devices, dip-molded gloves and balloons, asymmetric membranes, functional coatings, and as extruded profiles for cardiovascular catheters [3-5].
The properties of segmented polyurethanes depend on their structure, which is quite complicated. Their molecular structure is determined by the components used in the polymerization reaction and on stoichiometry, and they phase separate during the reaction forming a supermolecular structure [6-10]. Phase separation has been the subject of many studies $[11,12]$. The results showed that structural units of various forms and sizes develop during polymerization [13-15]. Besides a crystalline or at least highly ordered phase, soft and hard segments form corresponding phases which are partially soluble in

\footnotetext{
${ }^{*}$ Corresponding author, e-mail: bpukanszky@mail.bme.hu
} (c) BME-PT 
each other [16-21]. Solubility depends very much on the interaction of the components, the urethane groups are capable of forming specific interactions, $\mathrm{H}$-bonds with each other, but also with the soft segments $[22,23]$. Depending on preparation conditions, the final structure of the product is determined by kinetic effects, but a very large extent by interactions [24].

Polyurethanes are usually prepared from an isocyanate, a polyol and a chain extender [25]. Only a few isocyanates are used in practice, mostly the aromatic compounds 4,4'-methylenebis(phenyl isocyanate) (MDI) and toluene diisocyanate (TDI). The versatility of polyols is much larger, they may differ in chemical structure, molecular weight or functionality. Polyether and polyester polyols form probably the most important groups of this component $[1,25]$. It is obvious to assume that interactions will be different in polyurethanes prepared from the two kinds of polyols, and experience supports this assumption by showing that the properties of products prepared from them differ considerably [26]. Although the effect of the structure and characteristics of the polyol on PU properties have been investigated extensively [27-30], many questions related to structure-property correlations in these materials remain open.

The goal of this communication is to discuss the possible effect of specific interactions on the structure and properties of segmented linear polyurethane elastomers. Two series of samples were prepared from a polyether and a polyester polyol with similar molecular weight. The stoichiometric ratio of isocyanate and hydroxyl groups ( $\mathrm{NCO} / \mathrm{OH}$ ratio) was kept constant while the $-\mathrm{OH}$ functional group ratio of polyol/total diol ( $\mathrm{POH} / \mathrm{OH}$ ratio) was changed and used as variable in the experiments. Because of the larger molecular weight of the polyol compared to butanediol, the changing of this ratio results in a relative increase on the amount of the soft phase. Changing $\mathrm{POH} / \mathrm{OH}$ ratio makes possible the adjustment of properties (hardness, strength, elongation,
$T_{\mathrm{g}}$ ) to specific applications. Using the entire composition range allowed us seeing the effect of interactions and changing composition on a wider scale and also drawing more comprehensive conclusions even if the practical relevance of larger $\mathrm{POH} / \mathrm{OH}$ ratios is small. The structure of the polymers was characterized by various methods and an attempt was made to explain the apparently contradictory results with differences in interactions.

\section{Experimental}

4,4'-methylenebis(phenyl isocyanate) (MDI) was used as isocyanate and 1,4-butanediol (BD) as chain extender in both series. The polyether polyol was polytetrahydrofurane (PTHF), while the polyester was diol-end-capped poly(butanediol-adipate) (PBDA); both polyols had a molecular mass of $1000 \mathrm{~g} / \mathrm{mol}$. All the chemicals were acquired from Sigma-Aldrich Co. (USA). MDI was used as received, butanediol was distilled under vacuum at $190^{\circ} \mathrm{C}$ for 3 hours, and the polyols were dried at $80^{\circ} \mathrm{C}$ under vacuum for a day before the reaction. The stoichiometric ratio of isocyanate and hydroxyl groups $(\mathrm{NCO} / \mathrm{OH}$ ratio) was 1 in all materials. The variable was the $-\mathrm{OH}$ functional group ratio of polyol/total diol ( $\mathrm{POH} / \mathrm{OH}$ ratio), which changed from 0 to 1 in 0.1 steps. One step bulk polymerization was carried out in a W50EH internal mixer (Brabender GmbH \& Co., Duisburg, Germany) at $150^{\circ} \mathrm{C}, 50 \mathrm{rpm}$ for $30 \mathrm{~min}$. The polymer was compression molded into $1 \mathrm{~mm}$ plates at $200^{\circ} \mathrm{C}$ and 5 min using an SRA 100 (Fontijne Grotnes BV, Vlaardingen, Netherlands) machine. The molecular structure of the repeat units of the polyether and polyester polyurethane elastomers are shown in Figure 1.

The torque and temperature of mixing were recorded during polymerization. The time dependence of these quantities offers information about the kinetics of polymerization and about the molecular mass of the final product. Fourier transform attenuated total reflectance infra-red spectra (FTIR-ATR) were

a)

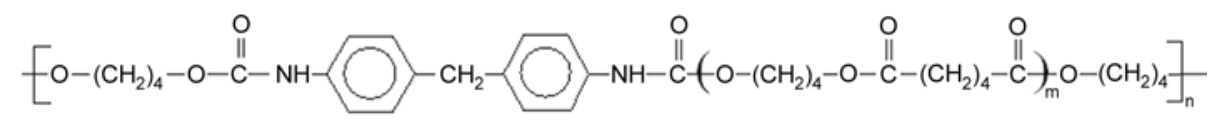

b) $\left[\mathrm{O}-\left(\mathrm{CH}_{2}\right)_{4}-\mathrm{O}-\mathrm{O} \mathrm{C}-\mathrm{NH}-\mathrm{O}-\mathrm{CH}_{2}-\mathrm{O}-\mathrm{NH}-\mathrm{C}\left(\mathrm{O}-\left(\mathrm{CH}_{2}\right)_{4}\right)_{\mathrm{m}}\right]_{\mathrm{n}}$

Figure 1. Molecular structure of the repeat units of the polyether and polyester polyurethane elastomers. a) polyester, b) polyether PU. 
recorded on the compression molded plates in the wavelength range of 4000 and $400 \mathrm{~cm}^{-1}$, using a Scimitar 2000 (Varian Inc., USA) apparatus equipped with a Specac Golden Gate ATR reflection unit and a wide band $\mathrm{Hg}-\mathrm{Cd}-\mathrm{Te}$ (MCT) detector. The original spectra were corrected before evaluation using the Advanced ATR Correction Algorithm by Thermo Scientific [31]. Specific interactions were estimated by the measurement of solvent absorption. Ethanol and n-octane were used as solvents and Flory-Huggins interaction parameters were calculated from equilibrium solvent uptake. The relaxation transitions of the various phases of the polymers were studied by differential scanning calorimetry (DSC) with a TA 4000 apparatus (Mettler Toledo, USA) equipped with a DSC 30 cell. Two heating and a cooling runs were done on $10 \mathrm{mg}$ samples with a rate of $20^{\circ} \mathrm{C} / \mathrm{min}$. Dynamic mechanical spectra (DMA) were recorded on samples with $20 \times 6 \times 1 \mathrm{~mm}$ dimensions between -120 and $200^{\circ} \mathrm{C}$ at $2{ }^{\circ} \mathrm{C} / \mathrm{min}$ heating rate in $\mathrm{N}_{2}$ atmosphere using a Pyris Diamond DMA apparatus (Perkin Elmer, USA). The measurements were carried out in tensile mode at $1 \mathrm{~Hz}$ frequency and $10 \mu \mathrm{m}$ deformation. The structure of the samples was characterized by X-ray diffraction (XRD). XRD patterns were recorded using a PW 1830/PW 1050 equipment (Phillips, Amsterdam, Netherlands) with $\mathrm{CuK}_{\alpha}$ radiation at $40 \mathrm{kV}$ and $35 \mathrm{~mA}$ in reflection mode. Mechanical properties were determined by tensile testing on dog-bone type specimens with $50 \times 10 \times 1 \mathrm{~mm}$ dimensions at $100 \mathrm{~mm} / \mathrm{min}$ cross-head speed using an 5566 (Instron, USA) apparatus. Tensile strength and elongation-at-break were derived from recorded force vs. elongation traces, while tensile modulus was determined from the initial, linear section of the traces. Shore A hardness was determined with an S1 protable durometer (Instron, USA) on $4 \mathrm{~mm}$ thick samples created by the stacking of $1 \mathrm{~mm}$ pieces. The transparency of the compression molded plates was measured using a Spekol ultra-violet-visible (UV-VIS) (Analytic, Jena, Germany) apparatus at $500 \mathrm{~nm}$ wavelength.

\section{Results and discussion}

The results of the study will be presented in several sections. The dependence of properties on composition, i.e. on the $\mathrm{POH} / \mathrm{OH}$ ratio, is shown first, followed by the characterization of structure. Specific interactions and their possible role in structure development are presented next, while practical consequences are discussed in the final section.

\subsection{Properties}

The molecular weight of all polymers should be the same, since the same stoichiometry, conditions and polyols with the same molecular weight are used for their preparation. Molecular weight can be determined by gel permeation chromatography (GPC) in dilute solution, but polyurethanes are not always easy to dissolve [32-33]. Since our polymers were prepared by reactive processing, the equilibrium torque measured during the reaction offers information about the molecular weight of the polymers produced. In an earlier study we found good agreement between molecular weight determined by GPC and torque recorded in the internal mixer [33]. Equilibrium torque determined for the two series is presented in Figure 2. Considerable differences can be seen in viscosity between the polyether and polyester polyurethanes in spite of our expectations. Several reasons may explain these differences. Under the conditions of reactive processing the polyols may have different reactivity leading to different kinetics and final molecular weights. Side reactions may also occur during polymerization and in different extents. However, detailed analysis of FTIR spectra could not reveal the formation of biuret or allophanate groups resulting from such reactions in

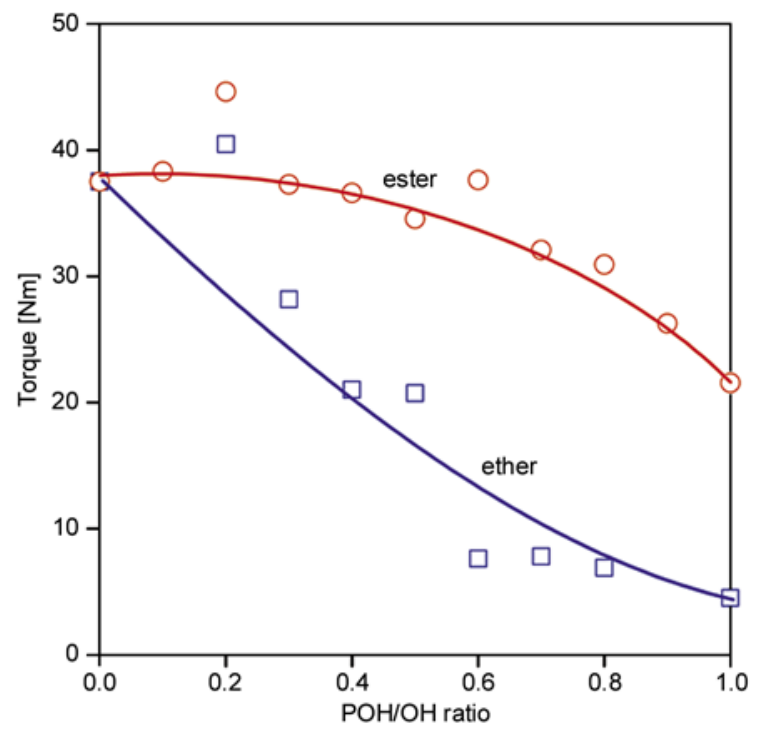

Figure 2. Effect of composition on the viscosity (equilibrium torque) measured at the end of the polymerization reaction in the mixer. Symbols: (o) polyester, ( $\square)$ polyether polyol. 
either of the series. Finally, we cannot exclude the role of interactions as a possible reason for the differences. The larger number of carboxyl groups in the polyester PU may interact with each other, but also with the urethane groups and increase viscosity. The decrease of viscosity with the $\mathrm{POH} / \mathrm{OH}$ ratio from 0 to 1 also indicates the role of interactions.

Polyurethane elastomers are often characterized by their Shore A hardness, which changes with the relative amount of polyol and chain extender. The hardness of the two series of polymers is compared to each other in Figure 3. Polyether polyurethanes are obviously softer than polyesters and the difference is more pronounced at larger $\mathrm{POH} / \mathrm{OH}$ ratios. We must assume that the phase segregated morphology differs in the two types of polymers and either the extent of phase separation or the properties of the phases change with composition. At small $\mathrm{POH} / \mathrm{OH}$ ratios hard segments (MDI and the chain extender) dominate structure, but with increasing polyol content the role of the chemical structure of the polyol, interactions and their effect on phase structure become more pronounced.

The tensile strength and deformability of PU elastomers are other important characteristics determining their possible application. These properties clearly depend both on the molecular and the phase structure of the polymer. In the absence of strong interactions molecular weight determines elongation and strength, while the formation of physical

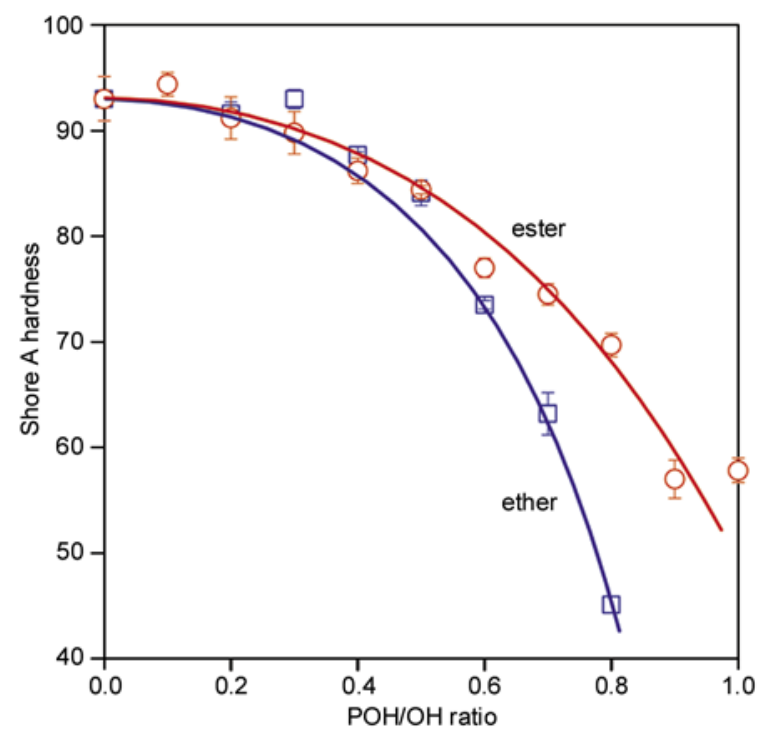

Figure 3. Changes in the Shore A hardness of segmented polyurethane elastomers with the $\mathrm{POH} / \mathrm{OH}$ ratio. Symbols: (o) polyester, $(\square)$ polyether polyol. cross-links results in a considerable increase in both properties. The tensile strengths of the two series are compared to each other in Figure 4. The difference is even larger than in the previous figure. Much larger strengths and different composition dependence is observed for the polyester urethanes than for their polyether counterparts. The maximum in the composition dependence of strength for the polyester PU is a result of several factors. With increasing polyol content the amount of soft phase increases, the size of the hard phase decreases and its distribution becomes more homogeneous. A larger number of smaller physical cross-link sites increase both elongation and strength (stain hardening). Because of weaker unlike interactions a smaller number of larger hard phase units form in the polyether PU and the effect of changing composition (increasing amount of soft phase) dominate (see also section 3.2. on structure). These differences may result from dissimilar molecular structure as suggested by the change of viscosity in Figure 2 or by different interactions and phase structure. The picture is further complicated by the results presented in Figure 5, in which the elongation-at-break values of the samples are plotted against the $\mathrm{POH} /$ $\mathrm{OH}$ ratio. The larger elongation of the polyether polyurethanes gives an indirect answer and proof that the molecular weight of the polymers is more or less the same. Ultimate elongation is proportional to molecular weight since larger molecules can uncoil more. The similar magnitude of elonga-

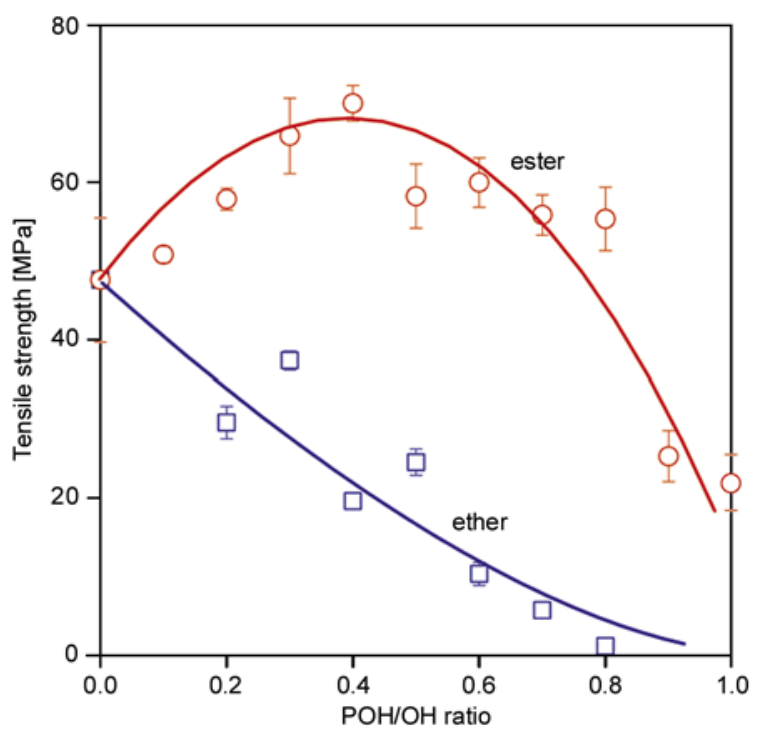

Figure 4. Composition dependence of the tensile strength of polyurethanes prepared with different polyols. Symbols: (o) polyester, ( $\square$ ) polyether polyol. 


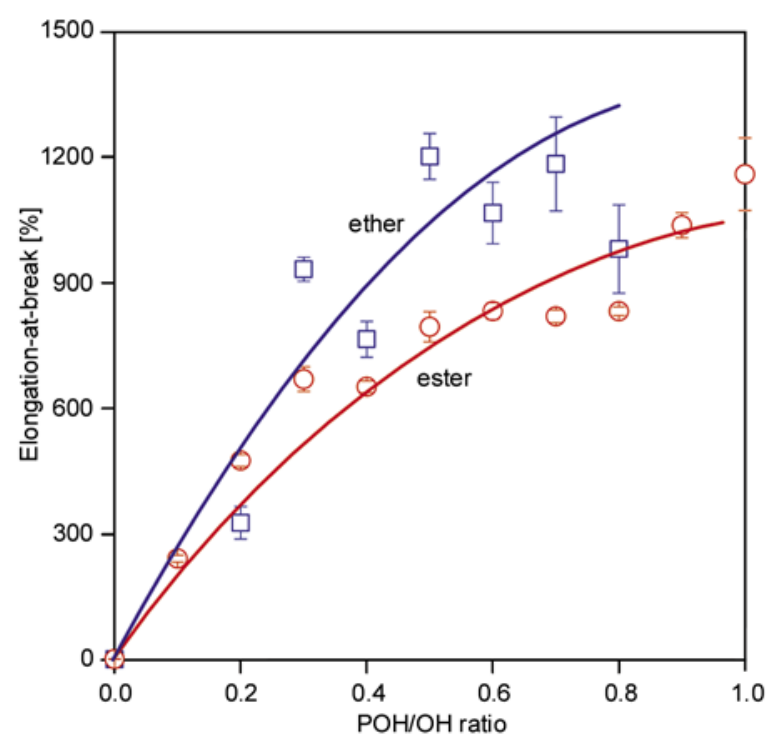

Figure 5. Ultimate elongation of polyurethane elastomers plotted against the $\mathrm{POH} / \mathrm{OH}$ ratio. Symbols: (o) polyester, ( $\square$ ) polyether polyol.

tions in the two series indicate similar molecular weights, while the smaller actual values of the polyester urethanes points to the role of interactions and phase structure. We may assume that polyether segments can enter into weaker interaction with each other and the urethane groups, thus less soft segments dissolve in the hard phase and the molecules remain more flexible. This leads to smaller hardness and larger elongation, but smaller strength. The results presented up to now emphasize the role of interactions and phase structure, thus we analyze structure in the next section.

\subsection{Structure}

Hard segments are known to form an ordered, crystalline phase in PU elastomers. This crystalline phase melts at high temperature and can be detected by DSC. Crystallinity and order is small, but this phase may hinder solubility and influence properties considerably. Besides DSC, another technique to study ordered, crystalline structures is X-ray diffraction. The XRD traces of selected polyurethanes are presented in Figure 6. Two compositions were selected for comparison, one rich in hard segments at $0.2 \mathrm{POH} / \mathrm{OH}$ ratio and one at high polyol content at $0.8 \mathrm{POH} / \mathrm{OH}$ ratio, where the soft phase dominates properties. We can see that order is clearly visible at small $\mathrm{POH} / \mathrm{OH}$ ratio, but the polymers are practically completely amorphous at large polyol content. Hardly any difference can be detected

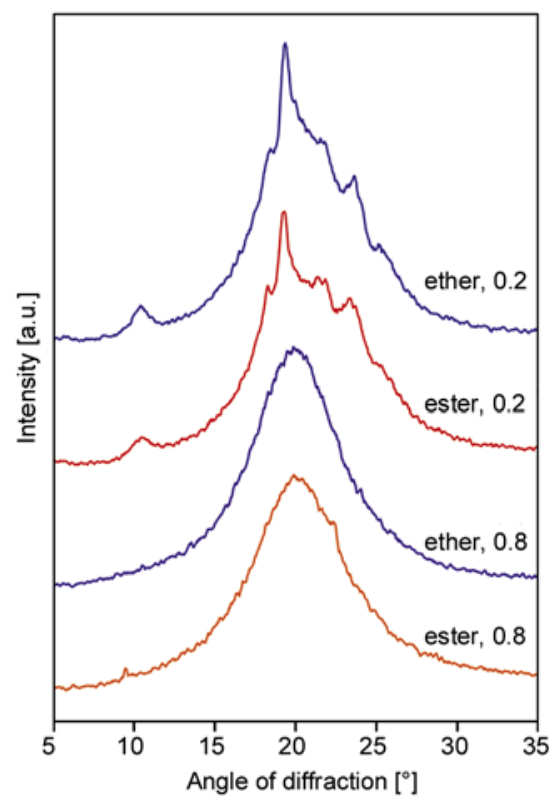

Figure 6. XRD traces of selected PU samples. Effect of composition on crystallinity. The number in the legend indicates the $\mathrm{POH} / \mathrm{OH}$ ratio.

between the polyurethanes prepared with the two types of polymers, but visual evaluation might be deceiving. The ether urethane seems to be more ordered than the ester at small $\mathrm{POH} / \mathrm{OH}$ ratio, while the polyester PU exhibits a very small peak at around 22.3 degree hinting at the presence of crystallinity. Unfortunately DSC traces did not confirm the presence of soft phase crystallinity, but this is not very surprising, since its amount is very small, if it is present at all. Quantitative analysis [34] of XRD spectra showed about $10 \%$ crystallinity for the polymer containing only hard segments, about $6 \%$ at a $\mathrm{POH} / \mathrm{OH}$ ratio of 0.2 and almost zero above $\mathrm{POH} /$ $\mathrm{OH}=0.4$. This result also means that not crystallinity, but interactions, and the structure and properties of the hard phase result in the differences in strength observed in Figure 4, since the polymers are practically completely amorphous in the composition range in which the strength of the two types of polymers differs the most $(\mathrm{POH} / \mathrm{OH}>0.4)$. Thermal methods offer valuable information about the phase structure of heterophase polymers and about the mobility of the segments going through transitions. The analysis of spectra may even offer some information about the composition of the phases. The DMA spectra of the two types of PU are compared to each other in Figure 7 at the $\mathrm{POH} / \mathrm{OH}$ ratio of 0.5 . We can see that the relaxation transition of the soft phase can be detected easily at sub ambi- 


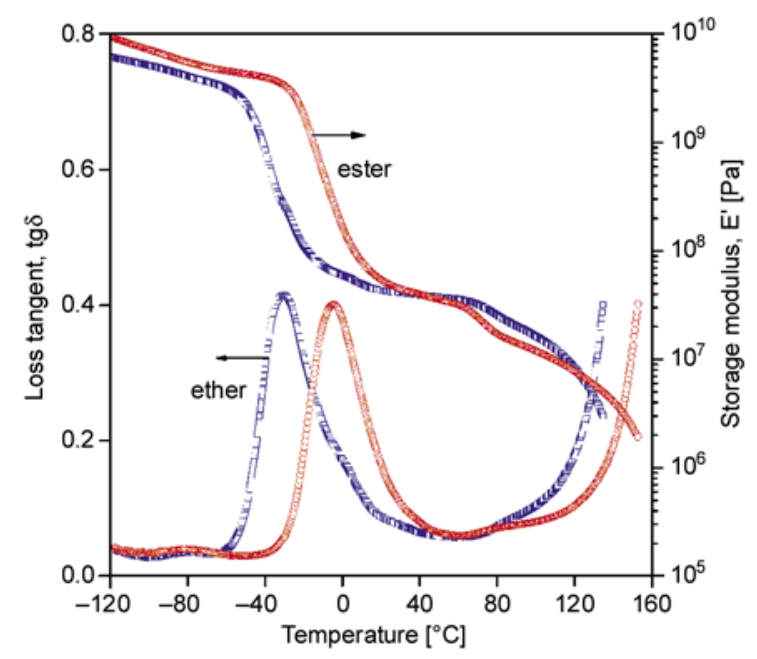

Figure 7 Comparison of the DMTA spectra of PU elastomers prepared with polyether and polyester polyols at $0.5 \mathrm{POH} / \mathrm{OH}$ ratio

ent temperatures. The soft phase of polyether urethane has lower glass transition temperature than that of the polyester PU. The detection and analysis of the hard phase is much more difficult. Hard segments are stiff and their mobility does not change much even after the transition. The transition can be detected by the change of the storage modulus, but identification is much more difficult on the $\tan \delta$ trace.

The analysis of DSC traces yields very similar results. The identification of the glass transition of the soft phase is unambiguous, while that of the hard phase is more difficult and the reliability of any quantity derived from the traces for the hard phase is much smaller. The composition dependence of the glass transition temperature of the phases determined by DSC is presented in Figure 8. and Table 1 . We can see that the $T_{\mathrm{g}}$ of both phases changes with composition. The transition temperature of the soft phase decreases continuously, while that of the hard phase drops quite considerably first, then increases slightly with increasing polyol content. We must call attention here to the fact that because of the difference in the molecular weight of butanediol and the polyol the amount of soft phase increases with increasing $\mathrm{POH} / \mathrm{OH}$ ratio thus leading to changes in partial solubility and in the composition of the phases. The drop in the $T_{\mathrm{g}}$ of the hard phase is a result of unlike interactions, which are stronger in the polyester than in the polyether PU. The slight increase at above the $\mathrm{POH} / \mathrm{OH}$ ration of 0.2 is probably caused by more pronounced phase

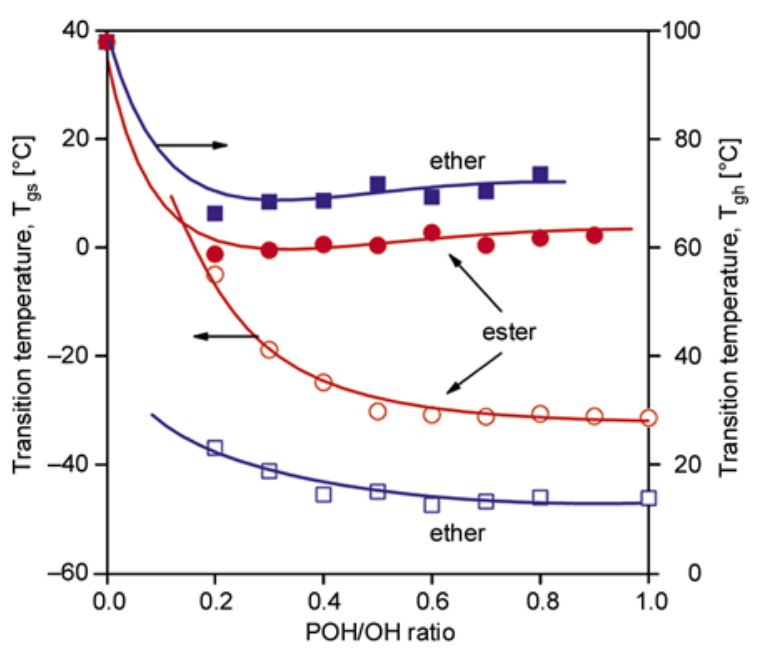

Figure 8. Changes in the glass transition temperature of the phases with composition. $T_{\mathrm{g}}$ was determined by $\mathrm{DSC}$ at $20^{\circ} \mathrm{C} / \mathrm{min}$ heating rate. Symbols: $(\mathrm{o}, \bullet)$ polyester, $(\square, \square)$ polyether polyol.

Table 1. Glass transition temperatures of the soft and hard phases of polyether and polyester polyurethanes elastomers determined by DSC

\begin{tabular}{|c|c|c|c|c|}
\hline \multirow{2}{*}{ POLI(OH)/OH } & \multicolumn{2}{|c|}{$\left.\mathbf{T}_{\text {gs }}{ }^{\circ} \mathbf{C}\right]$} & \multicolumn{2}{c|}{$\left.\mathbf{T}_{\text {gh }}{ }^{\circ} \mathbf{C}\right]$} \\
\cline { 2 - 5 } & ester & ether & ester & ether \\
\hline 0.0 & - & - & 97.89 & 97.89 \\
\hline 0.2 & -5.00 & -36.94 & 58.75 & 66.27 \\
\hline 0.3 & -18.85 & -41.21 & 59.48 & 68.43 \\
\hline 0.4 & -24.88 & -45.56 & 60.52 & 68.61 \\
\hline 0.5 & -30.22 & -45.02 & 60.36 & 71.68 \\
\hline 0.6 & -30.84 & -47.49 & 62.73 & 69.31 \\
\hline 0.7 & -31.20 & -46.84 & 60.41 & 70.32 \\
\hline 0.8 & -30.68 & -46.11 & 61.73 & 73.52 \\
\hline 0.9 & -31.10 & - & 62.22 & - \\
\hline 1.0 & -31.37 & -46.21 & - & - \\
\hline
\end{tabular}

separation with increasing soft segment content. The composition dependence of the transition temperature of the phases is similar for the two types of polymers, but the actual values are not. The $T_{\mathrm{g}}$ of the soft phase of polyester polyurethanes is larger in the entire composition range than in the polyether polymers, while the opposite is valid for the hard phase. These results indicate smaller mobility for the soft and larger for the hard segments in the polyester PU. Accordingly, we can conclude that the structural units of the polyester PU molecules enter into stronger interactions with each other than those in the polyether PU.

Further information is supplied about structure at a different scale by the dependence of light transmittance on composition (Figure 9). Large heterogeneities in a transparent matrix scatter light and 


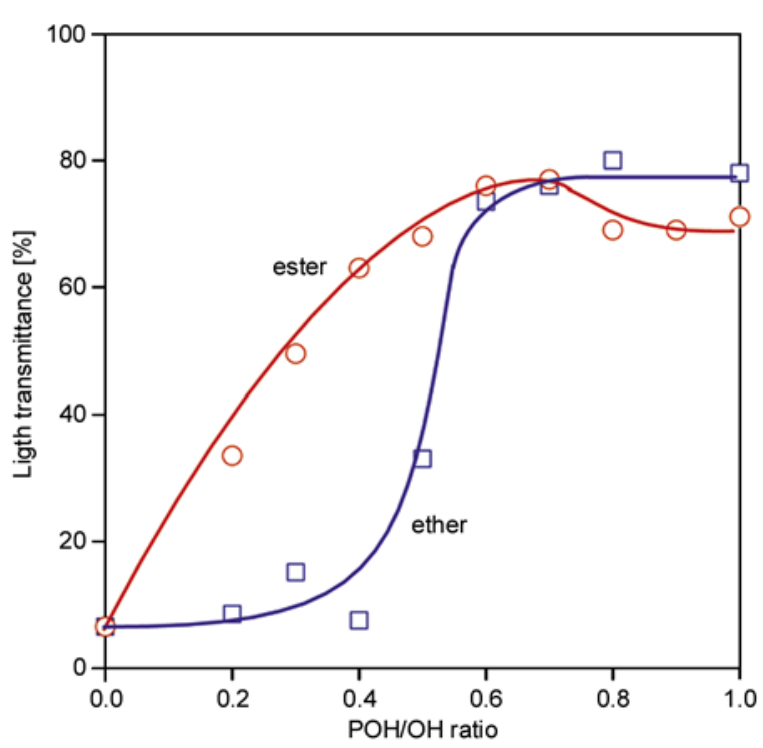

Figure 9. Effect of composition on the transparency of the polyurethane elastomers studied. Symbols: (o) polyester, $(\square)$ polyether polyol.

decrease the transparency of materials. Scattering starts for particles in the range of $20 \mathrm{~nm}$ and the material becomes completely opaque if the size of dispersed particles reaches several $100 \mathrm{~nm}$. At small $\mathrm{POH} / \mathrm{OH}$ ratios the composition dependence of transparency indicates large differences in the phase structure of the two types of polymers. The size of the dispersed phase decreases much faster in the polyester PU than in its polyether counterpart. The slight decrease of light transmittance above 0.8 polyol ratio might be related to the crystallization of the soft phase as indicated by XRD analysis (see Figure 6).

\subsection{Interactions}

As mentioned already, specific interactions are expected to develop between the carbonyl and ether groups of the polyol and the urethane groups, the former being stronger than the latter. Interactions may result in shifts in the corresponding vibrations in the FTIR spectra. Unfortunately the carbonyl groups of the polyester polyol absorb in the same wavelength range as the carbonyl in the urethane group. A detailed analysis of the spectra revealed only a substantial increase in the intensity of free carbonyl groups in the polyester PU as compared to the polyether polymer.

Another way to estimate interactions in a two phase polymer is the measurement of solvent absorption and the calculation of the Flory-Huggins interaction

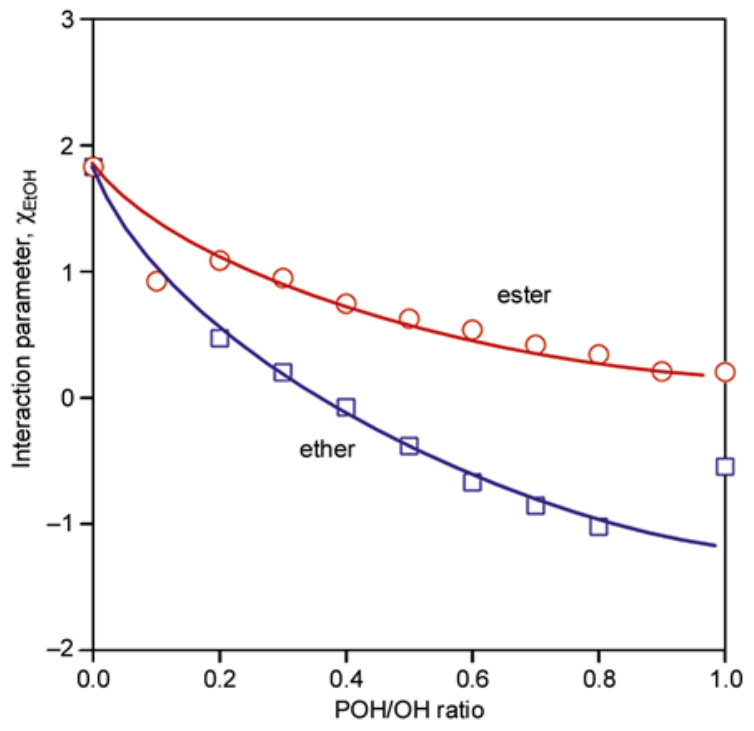

Figure 10. Flory-Huggins interaction parameter determined from ethanol absorption $\left(\chi_{\mathrm{EtOH}}\right)$ plotted as a function of $\mathrm{POH} / \mathrm{OH}$ ratio. Symbols: (o) polyester, ( $\square$ ) polyether polyol.

parameter from equilibrium solvent uptake. Interaction parameters determined with ethanol $\left(\chi_{\mathrm{EtOH}}\right)$ are plotted in Figure 10 as a function of composition. We can see that larger values are measured in the polyester polyurethane than in the polyether PU indicating weaker interaction. However, in order to understand competitive interactions in such a system, we must consider the interaction of all possible components. The $\chi_{\mathrm{ETOH}}$ values presented in Figure 10 represent the average interaction of the solvent molecules with the polymer and not the interaction between the segments of PU. Less solvent uptake indicates stronger self-interactions among the segments of the polymer leading to smaller solvent absorption and smaller interaction parameters. The results of these experiments confirm further our previous assumptions that unlike segments of the polyester PU form stronger interactions with each other than those of the polyether polymer.

Stronger interactions among the soft and hard segments should lead to smaller mobility of the soft segment and its larger partial solubility in the hard phase, as well as to decreased number of relaxing species. The number of relaxing units in a phase can be determined from the intensity of the transition, from the area or height of the $\tan \delta$ peak, or from the change in specific heat in the DSC trace. The intensity of soft phase transition is presented as a function of composition in Figure 11. The two types of 


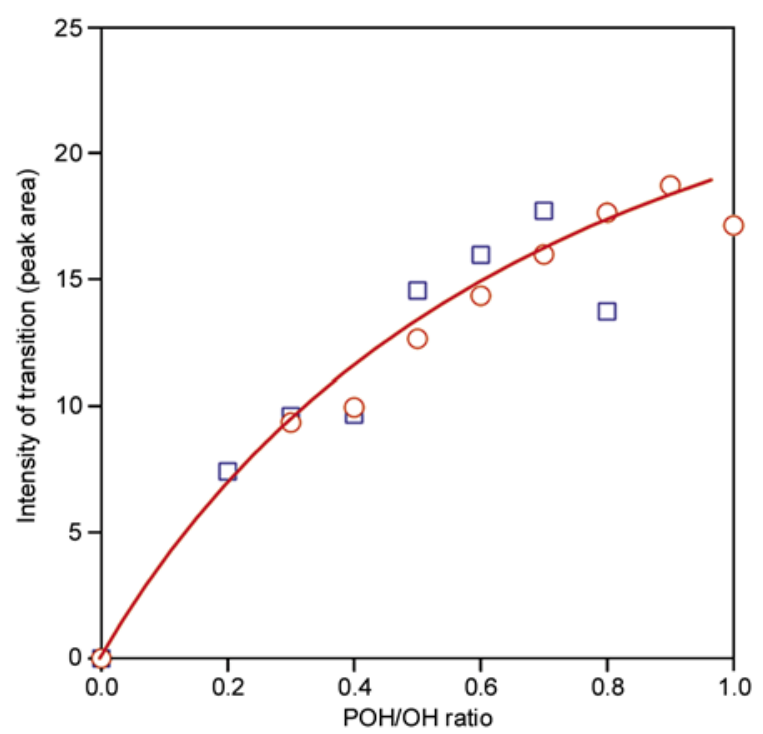

Figure 11. Effect of polyol type and composition on the amount of relaxing soft segments in polyurethane elastomers. Symbols: (o) polyester, ( $\square$ ) polyether polyol.

polymers obviously do not differ from each other in this respect that is rather surprising. Accepting the fact that the amount of the relaxing soft segments is approximately the same in the polyether and polyester urethanes, we must come to the conclusion that differences observed in properties must be caused by the size, number and characteristics of the hard phase.

\subsection{Discussion}

Results indicate that crystallinity or even order has limited importance at larger than $0.4 \mathrm{POH} / \mathrm{OH}$ ratio, but hard segments must play an important role in the determination of properties, especially in the large differences in strength observed in Figure 4. Dispersed units of the hard phase act as physical cross-links and determine the strength as well as deformability of the polymers. The difference in the density of physical cross-links, and/or their dissimilar properties are shown well by the strain hardening behavior of the polymers. Stress vs. strain traces of selected samples are presented in Figure 12. The tensile behavior of the two types of polymers differs significantly from each other. Much larger strengths are measured in the polyester polyurethanes at smaller ultimate deformations, than in the polyether samples, which can be explained with the larger number and more even distribution of physical cross-link sites. Larger hard phase units with

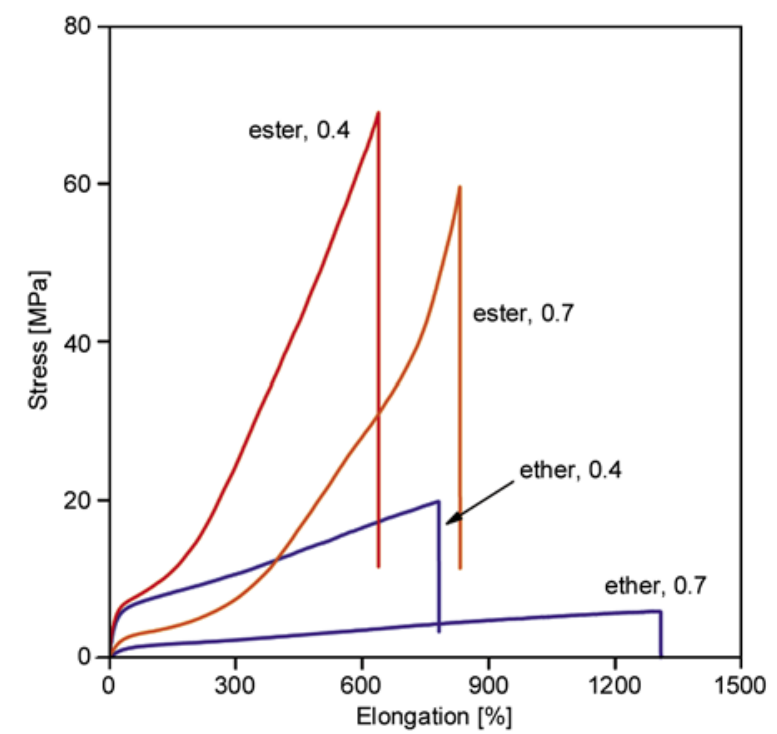

Figure 12. Stress vs. elongation traces of selected polyurethane elastomers; change in behavior with polyol type and composition. Numbers in the legend indicate $\mathrm{POH} / \mathrm{OH}$ ratio.

larger distances among them must result in the larger elongation of the polyether polyurethanes, and disentanglement of the chains and/or failure of these phases occurs at considerably smaller strengths. Obviously the stronger interaction of the polyester segments with the hard phase results in stronger materials.

The effect of physical cross-links can be visualized quite well if we calculate the work of deformation, i.e. if we multiply ultimate strength $(\sigma)$ with the corresponding elongation $\left(\Delta L / L_{0}\right)$, i.e. $\sigma \Delta L / L_{0}$. Crosslinking increases both strength and elongation up to a certain cross-link density in elastomers, and decreases above a critical value. The number and deformability of the soft segments is another important factor determining this quantity in our PU elastomers. The work of deformation is plotted against composition in Figure 13. We can see that the correlation has a maximum in both cases. The amount of the hard phase decreases continuously with increasing polyol content and the amount of soft phase thus also the mobility of the soft segments increases at the same time. At small $\mathrm{POH} / \mathrm{OH}$ ratio this leads to an increase in deformability, which together with the large number of homogeneously distributed physical cross-link sites increase the work of deformation. At very large $\mathrm{POH} / \mathrm{OH}$ ratios the number of cross-links decreases leading to a decrease in this characteristics. Although the ten- 


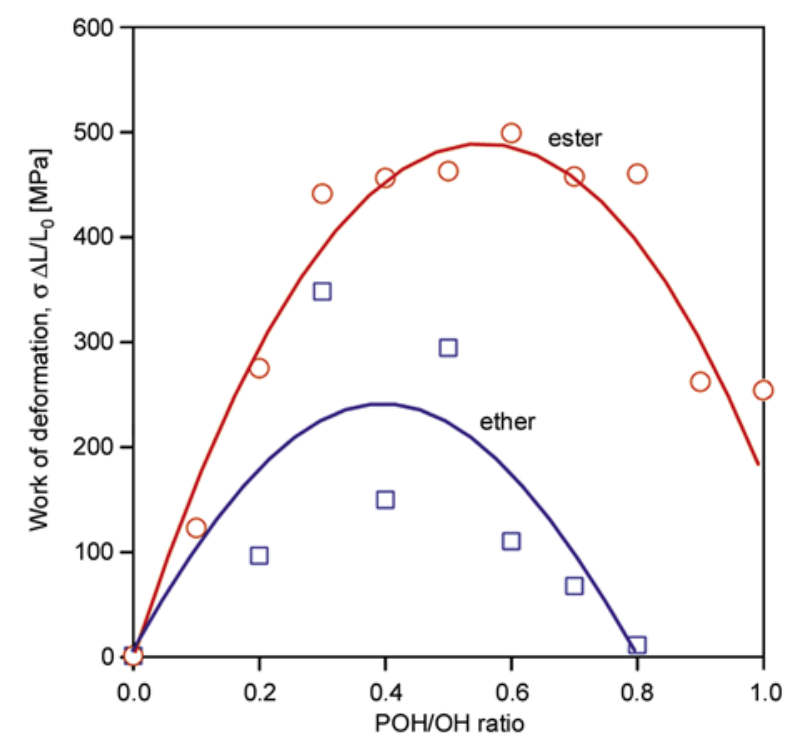

Figure 13. Work of deformation plotted against the $\mathrm{POH} /$ $\mathrm{OH}$ ratio of segmented polyurethane elastomers. Effect of polyol type on cross-link density. Symbols: (o) polyester, ( $\square$ ) polyether polyol.

dency is the same for the two polymers, the actual values, but also the scatter of the points differ considerably. Fewer cross-links distributed less homogeneously in the polyether polyurethane lead to smaller values and to a less unambiguous correlation. The strain hardening tendency of the polymers and additional analysis of mechanical properties clearly prove the role of interactions in the determination of the properties of segmented linear polyurethane elastomers.

\section{Conclusions}

The comparison of two sets of polyurethane elastomers prepared with a polyester and a polyether polyol of the same molecular weight proved that specific interactions determine the phase structure and properties of these materials. Crystallinity was approximately the same in the two types of polyurethanes and the amount of relaxing soft segments was also similar. The determination of interaction parameters from solvent absorption and differences in glass transition temperatures indicated stronger interaction between hard and soft segments in the polyester than in the polyether polyurethane. Larger transparency of the polyester PU indicated the formation of smaller dispersed particles of the hard phase. The larger number of smaller hard phase units led to the formation of more physical crosslinks distributed more evenly in the polymer. These differences in the phase structure of the polymers resulted in stronger strain hardening tendency, larger strength and smaller deformations for the polyester than for the polyether polyurethane.

\section{Acknowledgements}

The authors are indebted to Judith Mihály and Gábor Keresztury for their help in the recording and evaluation of FTIR-ATR spectra. The project was partially financed by the National Scientific Research Fund of Hungary (OTKA Grant No. K 68748) and by the New Hungary Development Plan (Project ID: TÁMOP-4.2.1/B-09/1/KMR-2010-0002); theirs support is highly appreciated. This work is connected to the scientific program of the 'Development of qualityoriented and harmonized $\mathrm{R}+\mathrm{D}+\mathrm{I}$ strategy and functional model at BME' project.

\section{References}

[1] Randall D., Lee S.: The polyurethanes book. Wiley, New York (2003).

[2] Seymour R. B., Kauffman G. B.: Polyurethanes: A class of modern versatile materials. Journal of Chemical Education, 69, 909-910 (1992).

DOI: 10.1021/ed069p909

[3] Zdrahala R. J., Zdrahala I. J.: Biomedical applications of polyurethanes: A review of past promises, present realities, and a vibrant future. Journal of Biomaterials Applications, 14, 67-90 (1999). DOI: $10.1177 / 088532829901400104$

[4] Lamba N. M. K., Woodhouse K. A., Cooper S. L.: Polyurethanes in biomedical applications. CRC Press, Boca Raton (1997).

[5] Bártolo P., Bidanda B.: Bio-materials and prototyping applications in medicine. Springer, New York (2008).

[6] Rausch K. W., Sayigh A. A. R.: Structure property relationships in polyurethane elastomers prepared by one-step reaction. Industrial and Engineering Chemistry Product Research and Development, 4, 92-98 (1965).

DOI: $10.1021 / 1360014 \mathrm{a} 008$

[7] Kojio K., Nakashima S., Furukawa M.: Microphaseseparated structure and mechanical properties of norbornane diisocyanate-based polyurethanes. Polymer, 48, 997-1004 (2007). DOI: 10.1016/j.polymer.2006.12.057

[8] Velankar S., Cooper S. L.: Microphase separation and rheological properties of polyurethane melts. 1. Effect of block length. Macromolecules, 31, 9181-9192 (1998).

DOI: $10.1021 / \mathrm{ma9811472}$

[9] Velankar S., Cooper S. L.: Microphase separation and rheological properties of polyurethane melts. 2. Effect of block incompatibility on the microstructure. Macromolecules, 33, 382-394 (2000).

DOI: $10.1021 / \mathrm{ma} 990817 \mathrm{~g}$ 
[10] Velankar S., Cooper S. L.: Microphase separation and rheological properties of polyurethane melts. 3. Effect of block incompatibility on the viscoelastic properties. Macromolecules, 33, 395-403 (2000).

DOI: $10.1021 / \mathrm{ma} 9908189$

[11] Furukawa M., Mitsui Y., Fukumaru T., Kojio K.: Microphase-separated structure and mechanical properties of novel polyurethane elastomers prepared with ether based diisocyanate. Polymer, 46, 10817-10822 (2005).

DOI: 10.1016/j.polymer.2005.09.009

[12] Pompe G., Pohlers A., Pötschke P., Pionteck J.: Influence of processing conditions on the multiphase structure of segmented polyurethane. Polymer, 39, 51475153 (1998).

DOI: $10.1016 / \mathrm{S} 0032-3861(97) 10350-0$

[13] Tocha E., Janik H., Debowski H., Vancso G. J.: Morphology of polyurethanes revisited by complementary AFM and TEM. Journal of Macromolecular Science Part B: Physics, 41, 1291-1304 (2002).

DOI: $10.1081 / \mathrm{MB}-120013098$

[14] Król P., Pilch-Pitera B.: Phase structure and thermal stability of crosslinked polyurethane elastomers based on well-defined prepolymers. Journal of Applied Polymer Science, 104, 1464-1474 (2007).

DOI: $10.1002 / a p p .25011$

[15] Garrett J. T., Xu R., Cho J., Runt J.: Phase separation of diamine chain-extended poly(urethane) copolymers: FTIR spectroscopy and phase transitions. Polymer, 44, 2711-2719 (2003).

DOI: $10.1016 / \mathrm{S} 0032-3861(03) 00165-4$

[16] Li Y., Kang W., Stoffer J. O., Chu B.: Effect of hardsegment flexibility on phase separation of segmented polyurethanes. Macromolecules, 27, 612-614 (1994). DOI: $10.1021 / \mathrm{ma} 00080 \mathrm{a} 043$

[17] Chu B., Gao T., Li Y., Wang J., Desper C. R., Byrne C. A.: Microphase separation kinetics in segmented polyurethanes: Effects of soft segment length and structure. Macromolecules, 25, 5724-5729 (1992).

DOI: $10.1021 / \mathrm{ma} 00047 \mathrm{a} 025$

[18] Miller J. A., Lin S. B., Hwang K. K. S., Wu K. S., Gibson P. E., Cooper S. L.: Properties of polyether-polyurethane block copolymers: Effects of hard segment length distribution. Macromolecules, 18, 32-44 (1985) DOI: $10.1021 / \mathrm{ma} 00143 \mathrm{a} 005$

[19] Saiani A., Daunch W. A., Verbeke H., Leenslag J-W., Higgins J. S.: Origin of multiple melting endotherms in a high hard block content polyurethane. 1 . Thermodynamic investigation. Macromolecules, 34, 90599068 (2001).

DOI: $10.1021 / \mathrm{ma} 0105993$

[20] Saiani A., Rochas C., Eeckhaut G., Daunch W. A., Leenslag J-W., Higgins J. S.: Origin of multiple melting endotherms in a high hard block content polyurethane. 2. Structural investigation. Macromolecules, 37, 1411-1421 (2004).

DOI: $10.1021 / \mathrm{ma} 034604+$
[21] Saiani A., Novak A., Rodier L., Eeckhaut G., Leenslag J-W., Higgins J. S.: Origin of multiple melting endotherms in a high hard block content polyurethane: Effect of annealing temperature. Macromolecules, 40, 7255-7262 (2007).

DOI: $10.1021 / \mathrm{ma} 070332 \mathrm{p}$

[22] Yilgör E., Burgaz E., Yurtsever E., Yilgör I.: Comparison of hydrogen bonding in polydimethylsiloxane and polyether based urethane and urea copolymers. Polymer, 41, 849-857 (2000).

DOI: $10.1016 / \mathrm{S} 0032-3861(99) 00245-1$

[23] Liu J., Ma D., Li Z.: FTIR studies on the compatibility of hard-soft segments for polyurethane-imide copolymers with different soft segments. European Polymer Journal, 38, 661-665 (2002).

DOI: $10.1016 / \mathrm{S} 0014-3057(01) 00247-6$

[24] Król P.: Synthesis methods, chemical structures and phase structures of linear polyurethanes. Properties and applications of linear polyurethanes in polyurethane elastomers, copolymers and ionomers. Progress in Materials Science, 52, 915-1015 (2007).

DOI: $10.1016 /$ j.pmatsci.2006.11.001

[25] Macosko C.W.: RIM, Fundamentals of reaction injection molding. Hanser, Munich (1989).

[26] Crawford D. M., Bass R. G., Haas T. W.: Strain effects on thermal transitions and mechanical properties of thermoplastic polyurethane elastomers. Thermochimica Acta, 323, 53-63 (1998). DOI: 10.1016/S0040-6031(98)00541-3

[27] Sonnenschein M. F., Lysenko Z., Brune D. A., Wendt B. L., Schrock A. K.: Enhancing polyurethane properties via soft segment crystallization. Polymer, 46, 10158-10166 (2005).

DOI: $10.1016 /$ j.polymer.2005.08.006

[28] Eceiza A., de la Caba K., Kortaberria G., Gabilondo N., Marieta C., Corcuera M. A., Mondragon I.: Influence of molecular weight and chemical structure of soft segment in reaction kinetics of polycarbonate diols with 4,4'-diphenylmethane diisocyanate. European Polymer Journal, 41, 3051-3059 (2005). DOI: $10.1016 /$ j.eurpolymj.2005.06.022

[29] Hernandez R., Weksler J., Padsalgikar A., Choi T., Angelo E., Lin J. S., Xu L-C., Siedlecki C. A., Runt J.: A comparison of phase organization of model segmented polyurethanes with different intersegment compatibilities. Macromolecules, 41, 9767-9776 (2008).

DOI: $10.1021 / \mathrm{ma} 8014454$

[30] Rueda-Larraz L., d'Arlas F. B., Tercjak A., Ribes A., Mondragon I., Eceiza A.: Synthesis and microstructure-mechanical property relationships of segmented polyurethanes based on a PCL-PTHF-PCL block copolymer as soft segment. European Polymer Journal, 45, 2096-2109 (2009).

DOI: $10.1016 /$ j.eurpolymj.2009.03.013 
[31] Stangret J., Kamienska-Piotrowicz E., Laskowska K.: FT-IR studies of molecular interactions in formamidemethanol mixtures. Vibrational Spectroscopy, 44, 324 330 (2007).

DOI: $10.1016 /$ j.vibspec.2007.02.003

[32] Ojha U., Kulkarni P., Faust R.: Syntheses and characterization of novel biostable polyisobutylene based thermoplastic polyurethanes. Polymer, 50, 3448-3457 (2009).

DOI: $10.1016 /$ j.polymer.2009.05.025
[33] Pukánszky B. Jr., Bagdi K., Tóvölgyi Z., Varga J., Botz L., Hudak S., Dóczi T., Pukánszky B.: Nanophase separation in segmented polyurethane elastomers: Effect of specific interactions on structure and properties. European Polymer Journal, 44, 2431-2438 (2008).

DOI: $10.1016 /$ j.eurpolymj.2008.06.008

[34] Brückner S.: Estimation of the background in powder diffraction patterns through a robust smoothing procedure. Journal of Applied Crystallography, 33, 977-979 (2000).

DOI: $10.1107 / \mathrm{S} 0021889800003617$ 